\title{
Differential roles of Stella in the modulation of DNA methylation during oocyte and zygotic development
}

\author{
Longsen Han', Chao Ren², Jun Zhang ${ }^{1}$, Wenjie Shu (1) ${ }^{2}$ and Qiang Wang (1)
}

Dear Editor,

Stella (also known as PGC7 or Dppa3) was identified as a highly expressed protein in primordial germ cells (PGC). Expression of Stella is maintained throughout oocyte maturation and persists into the preimplantation embryos $^{1}$. Stella knockout females display a strongly reduced fertility due to the impaired developmental competence of early embryos ${ }^{2}$. The potential involvement of Stella in embryogenesis and generation of induced pluripotent stem (iPS) cells has been examined ${ }^{3,4}$. However, the effects of Stella on oocyte development, especially at cellular and molecular level, remain unknown. To do this, we generated Stella mutant mice using the CRISPR/Cas9 system (Supplementary Fig. S1a). Mice carrying homozygous mutations were born alive in a knockout line containing 153-bp deletion $\left(\mathrm{Stella}^{\Delta}\right)$. We confirmed the successful deletion of Stella in the mutant mice by western blot analysis (Supplementary Fig. S1b). Stella $^{\Delta}$ females were infertile when crossed with mutant males despite the ovulation of eggs with normal appearance (Supplementary Fig. S1c-e). After fertilization, Stella $^{\Delta}$ embryos are compromised in the preimplantation development and rarely reach the blastocyst stage (Supplementary Fig. S1f-g), consistent with published data ${ }^{2}$. It is worth noting that this truncated protein was absent in the nucleus, but was still detectable in the cytoplasm of Stella $^{\Delta}$ zygotes based on immunostaining (Supplementary Fig. S2, arrows). Recently, Shin et al., revealed that maternal Stella is partially cleaved by the ubiquitin-

Correspondence: Wenjie Shu (shuwj@bmi.ac.cn) or

Qiang Wang (qwang2012@njmu.edu.cn)

${ }^{1}$ State Key Laboratory of Reproductive Medicine, Nanjing Medical University, Nanjing, Jiangsu 211166, China

2Department of Biotechnology, Beijing Institute of Radiation Medicine, Beijing 100850, China

These authors contributed equally: Longsen Han, Chao Ren. proteasome system and an $\mathrm{N}$-terminal fragment remains in the cytoplasm where it participates in vesicular trafficking ${ }^{5}$. Therefore, our mutant mouse model may provide novel insights into Stella function compared to the conventional knockout mice reported previously ${ }^{6,7}$.

Given that Stella modulates the epigenetic asymmetry in zygotes, we asked whether Stella is also involved in the establishment of DNA methylation during oogenesis. To address this question, ovulated oocytes from Stella ${ }^{\Delta}$ and wild-type (WT) mice were isolated, and then baseresolution methylomes were generated using the bisulfite sequencing (BS-Seq) method for small samples (Fig. 1a). We found that, in WT oocytes, the global DNA methylation level was $\sim 38 \%$, as expected ${ }^{8}$. However, in Stella $^{\Delta}$ oocytes, the average methylation level was dramatically increased to $\sim 68 \%$ (Fig. $1 \mathrm{~b}-\mathrm{c}$ ). This extensive elevation of DNA methylation was observed across all genomic features examined, such as promoter, untranslated region (UTR), CpG island (CGI), intron, exon, as well as the major repetitive-elements (Fig. 1d-g; Supplementary Fig. S3). Such a pattern indicates that the changes in DNA methylation of Stella $^{\Delta}$ oocytes are in general universal throughout the entire genome. To gain a better understanding of the altered methylation landscape, we also conducted a search for differentially methylated regions (DMRs) between WT and Stella ${ }^{\Delta}$ oocytes. In total, 21,036 DMRs were identified, of which 20,998 were hypermethylated (hyper-DMRs; 99.8\%) and only 38 were hypomethylated (hypo-DMRs; 0.2\%) (Fig. 1h; Supplementary Table S1), showing a predominance of hyperDMRs. In the female germline, de novo methylation takes place during the postnatal growth phase of oocytes. Stella was shown to be able to inhibit recruitment of the DNA methyltransferase, DNMT1, through the binding of UHRF $1{ }^{9}$, which might be the critical pathway mediating

\section{(c) The Author(s) 2019}

(c) (i) Open Access This article is licensed under a Creative Commons Attribution 4.0 International License, which permits use, sharing, adaptation, distribution and reproduction in any medium or format, as long as you give appropriate credit to the original author(s) and the source, provide a link to the Creative Commons license, and indicate if changes were made. The images or other third party material in this article are included in the article's Creative Commons license, unless indicated otherwise in a credit line to the material. If material is not included in the article's Creative Commons license and your intended use is not permitted by statutory regulation or exceeds the permitted use, you will need to obtain permission directly from the copyright holder. To view a copy of this license, visit http://creativecommons.org/licenses/by/4.0/. 


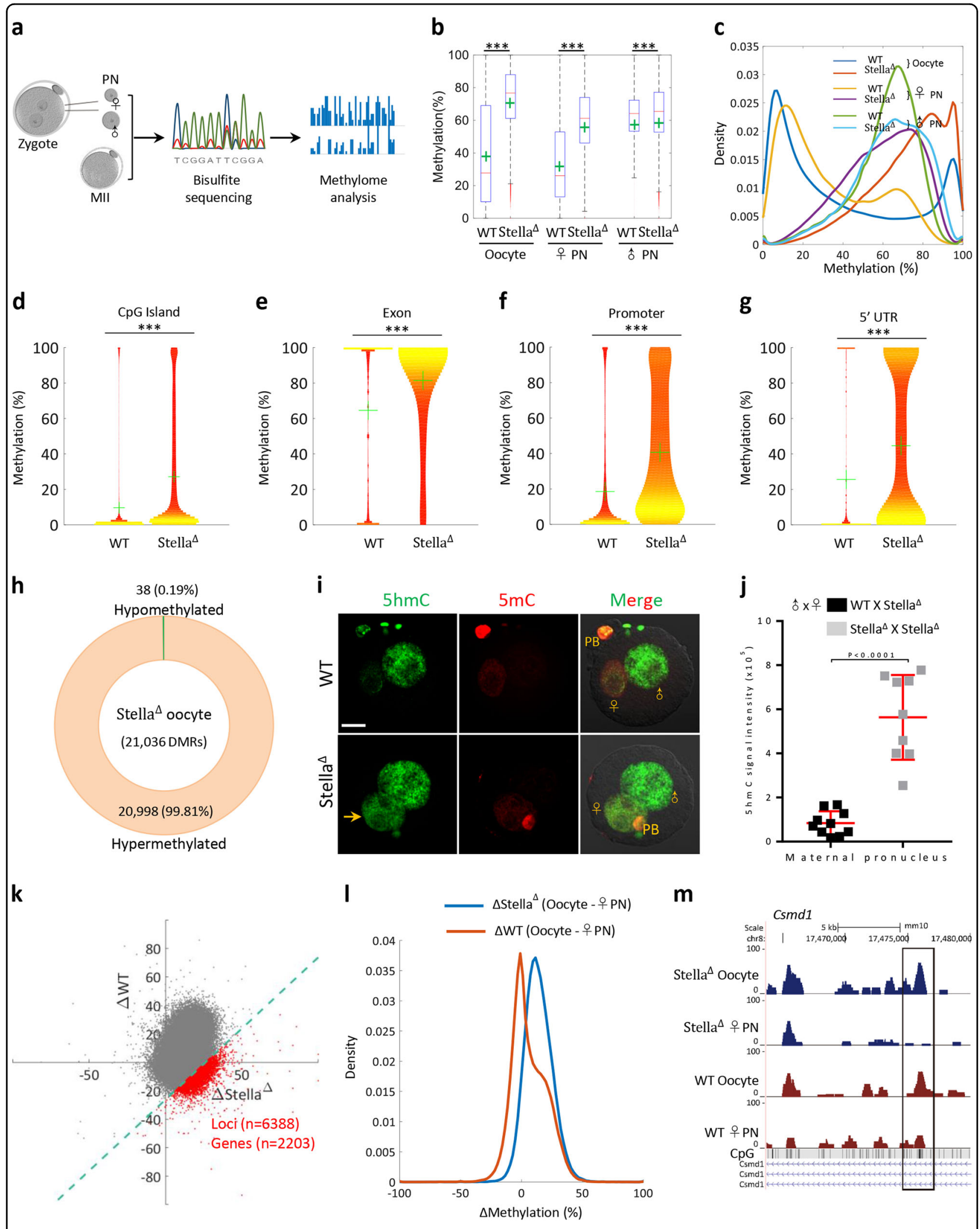

Fig. 1 (See legend on next page.) 
(see figure on previous page)

Fig. 1 Differing roles of Stella in the control of DNA methylation during oocyte and zygotic development. a Diagram illustrating the BS-seq procedure for genome-wide methylation analysis. Individual parental pronuclei and ovulated oocytes were collected, and DNA was bisulfite converted, followed by library preparation and high-throughput sequencing. b Distribution of the average methylation level across 20-kb windows in oocyte, female pronucleus (PPN), male pronucleus( ${ }^{\mathrm{A} P N}$ ) from Stella ${ }^{\Delta}$ and WT mice. Boxplot illustrates the median (red bar), mean (green cross), 25/75 percentage range (box), maximum and minimum (whiskers), and extreme values (red dots outside box). c Density plot of the average methylation level across 20-kb windows in oocyte, oPN, ${ }^{\mathrm{T} P N}$ from Stella ${ }^{\Delta}$ and WT mice. $\mathbf{d}-\mathbf{g}$ Violin plots show the methylation levels for four genomic features in oocytes from Stella ${ }^{\Delta}$ and WT mice. The green cross indicates the mean methylation levels. Bootstrap test was used to for statistical analysis. $\mathbf{h}$ Total number of DMRs identified between oocyte from Stella ${ }^{\Delta}$ and WT mice. The proportions of hyper- and hypo-DMRs are presented in circular plot. i PN4 zygotes from WT and Stella ${ }^{\Delta}$ mice were stained with anti-5mC (red) and anti-5hmC (green) antibodies. Arrows indicate the gain of $5 \mathrm{hmC}$ in the maternal PN of Stella ${ }^{\Delta}$ zygotes. PB, polar body. Scale bar, $20 \mu \mathrm{m}$. j Quantification of $5 \mathrm{hmC}$ fluorescence intensity in female pronuclei of zygotes. Each data point represents one maternal PN in zygotes ( $n=10$ for each group). A Student's $t$-test was used for statistical analysis. $\mathbf{k}$ Scatter plot illustrates the difference in DNA methylation level across 20-kb windows in the groups as indicated. $X$-axis represents the difference in DNA methylation level between oocyte and PN from Stella ${ }^{\Delta}$ mice, and $Y$-axis represents the difference in DNA methylation level between oocyte and $\$ P N$ from WT mice. The red dots denote the $20-\mathrm{kb}$ windows with large difference $(<5 \%)$ in DNA methylation level in Stella ${ }^{\Delta}$ group compared with WT group. Green line $(X-Y=26.31)$ indicates the threshold corresponding to $5 \%$. I Density plot of the difference in average DNA methylation level across $20-k b$ windows between oocyte and PPN from Stella ${ }^{\Delta}$ and WT mice. $\mathbf{m}$ Screenshot of Csmd1 gene, as an example of a gene whose methylation is protected by Stella following fertilization

the effects of Stella on methylation landscape in oocytes. Together, our findings clearly suggest that Stella is a novel and essential factor preventing excessive DNA methylation during oocyte development.

To track the effects of Stella on the DNA methylation of parental genome, individual female and male pronuclei of late-stage zygotes were isolated separately for genomewide profiling (Fig. 1a). Limited change was observed in the methylation levels of maternal DNA between oocytes and zygotes from WT mice, as expected ${ }^{8}$. However, maternal DNA methylome is markedly demethylated from oocytes $(68 \%)$ to zygotes $(55 \%)$ in Stella ${ }^{\Delta}$ mice (Fig. 1b-c). In support of this, we found a significant increase in $5 \mathrm{hmC}$ signal in the female pronuclei of Stella ${ }^{\Delta}$ zygotes; whereas female pronuclei in WT zygotes showed a much less intense $5 \mathrm{hmC}$ staining (Fig. 1i-j). These results strongly indicate the active demethylation of maternal genome during Stella ${ }^{\Delta}$ zygote development, supportive of the model proposed by Wang et al., Nakamura et al., and Armouroux et al. ${ }^{8,10}$. TET3 is a critical dioxygenase that catalyzes conversion of $5 \mathrm{mC}$ to $5 \mathrm{hmC}$ in the paternal genome, while Stella could block TET3 activity to maintain DNA methylation of the maternal $\mathrm{DNA}^{4,7}$. Heavy demethylation of maternal genome in Stella $^{\Delta}$ zygotes was likely due to the TET3 gaining access to female pronuclei. Next, in order to search for those potential loci and genes whose methylation is protected by Stella, we analyzed the difference in DNA methylation level of $20 \mathrm{~kb}$ windows between oocyte and maternal pronuclei from WT and Stella ${ }^{\Delta}$ mice, respectively. Totally, 6388 genomic loci and 2203 genes were identified (Fig. 1k-l; Supplementary Table S2), such as the development-related genes Csmd1 (Fig. 1m), Zdhhc6, and Erbb4 (Supplementary Fig. S4). Gene ontology (GO) analysis further indicates that these genes are enriched in the pathways that play important roles in nervous system and metabolic process (Supplementary Fig. S4).
Interestingly, compared to WT zygotes, the average methylation level of paternal genome in Stella ${ }^{\Delta}$ zygote was increased significantly, although not dramatically (Fig. 1b-c). More assays are needed to clarify this issue. Cumulatively, our findings suggest that Stella participates in the DNA methylation maintenance of maternal genome during mouse zygotic development.

On the other hand, we noticed that, although the DNA demethylation has occurred, the average methylation level of female pronuclei was still elevated in Stella ${ }^{\Delta}$ zygotes when compared to WT zygotes (Fig. 1b-c). This observation prompted us to propose that such a high level of maternal DNA methylation in Stella ${ }^{\Delta}$ zygotes was likely originated from the global hypermethylation in oocytes. To test this possibility, we evaluated the contribution of different genomic features to the DNA hypermethylation in Stella ${ }^{\Delta}$ oocytes and zygotes. Gain of methylation was detected in all elements of Stella ${ }^{\Delta}$ oocytes, with the largest proportion contributed by intergenic region (48\%; Supplementary Fig. S5a-b). Maternal genome of Stella ${ }^{\Delta}$ zygotes displayed the similar pattern compared with their WT counterparts (Supplementary Fig. S5a-b). Of note, the extent of demethylation of distinct elements was almost identical between oocytes and female pronuclei in either WT or Stella ${ }^{\Delta}$ mice, as evidenced by similar methylation patterns (Supplementary Fig. S5b-c). This observation indicates that Stella has no preference for the specific genomic regions when protecting maternal DNA against demethylation in zygotes. Moreover, we found that $91 \%$ of genes with hyper-DMR (621/680) identified in maternal genome of Stella ${ }^{\Delta}$ zygotes were indeed inherited from their oocytes (Supplementary Fig. S6). Altogether, these data suggest that global hypermethylation across the oocyte methylome in Stella ${ }^{\Delta}$ mice results in the higher level of DNA methylation in female pronuclei than that in WT mice. Considering that BS-Seq measures the sum of $5 \mathrm{mC}$ and $5 \mathrm{hmC}$, and the strong $5 \mathrm{hmC}$ signals were 
detectable in Stella ${ }^{\Delta}$ female pronuclei, more dramatic DNA demethylation perhaps occurred during the transition from oocytes to zygote in Stella ${ }^{\Delta}$ mice.

In summary, by constructing a Stella mutant mouse model, we identified Stella as a novel factor essential for preventing excessive DNA methylation during oogenesis. Following fertilization, Stella participates in the maintenance of maternal genome methylation during zygotic development. After we submitted this manuscript, Li et al. reported the function of Stella in safeguarding the oocyte methylome $^{11}$, further supporting our conclusion. Our work also provides a comprehensive atlas at the genomewide scale of the DNA methylation landscape in oocytes and zygotes from Stella ${ }^{\Delta}$ mice, which offers new insights into the Stella function in epigenetic control.

\section{Acknowledgements}

We are grateful to Dr. Qi Chen (University of Nevada) for his valuable discussions and critical reading of the manuscript. This work was supported by the National Key Research and Development Program (2017YFC1001500 to Q.W.), National Natural Science Foundation (No. 31771657 and 31571543), the Science Foundation for Distinguished Young Scholars of Jiangsu Province (BK20180035 to Q.W.), Major Research Plan of the National Natural Science Foundation (U1435222 to W.S.), Major Research Plan of the National Key R\&D Program (2016YFC0901600 to W.S.) of China.

\section{Author contributions}

L.H. and Q.W. conceived the projects. L.H. and J.Z. performed the experiments on mouse model and embryo manipulation. C.R. and W.S. contributed to the sequencing and bioinformatics analysis. L.H., C.R., W.S., and Q.W. wrote the manuscript.

\section{Conflict of interest}

The authors declare that they have no conflict of interest.

\section{Publisher's note}

Springer Nature remains neutral with regard to jurisdictional claims in published maps and institutional affiliations.

Supplementary Information accompanies the paper at (https://doi.org/ 10.1038/s41421-019-0081-2).

Received: 28 November 2018 Revised: 3 January 2019 Accepted: 4 January 2019

Published online: 29 January 2019

\section{References}

1. Sato, M. et al. Identification of PGC7, a new gene expressed specifically in preimplantation embryos and germ cells. Mech. Dev. 113, 91-94 (2002).

2. Payer, B. et al. Stella is a maternal effect gene required for normal early development in mice. Curr. Biol. 13, 2110-2117 (2003).

3. $\mathrm{Xu}, \mathrm{X}$. et al. Dppa3 expression is critical for generation of fully reprogrammed iPS cells and maintenance of Dlk1-Dio3 imprinting. Nat. Commun. 6, 6008 (2015).

4. Nakamura, T. et al. PGC7 binds histone H3K9me2 to protect against conversion of $5 \mathrm{mC}$ to $5 \mathrm{hmC}$ in early embryos. Nature $\mathbf{4 8 6}, 415-419$ (2012).

5. Shin, S. W., Vogt, E. J., Jimenez-Movilla, M., Baibakov, B. \& Dean, J. Cytoplasmic cleavage of DPPA3 is required for intracellular trafficking and cleavage-stage development in mice. Nat. Commun. 8, 1643 (2017).

6. Bortvin, A., Goodheart, M., Liao, M. \& Page, D. C. Dppa3 / Pgc7 / stella is a maternal factor and is not required for germ cell specification in mice. BMC Dev. Biol. 4,2 (2004).

7. Nakamura, T. et al. PGC7/Stella protects against DNA demethylation in early embryogenesis. Nat. Cell Biol. 9, 64-71 (2007).

8. Wang, L. et al. Programming and inheritance of parental DNA methylomes in mammals. Cell 157, 979-991 (2014).

9. Funaki, S. et al. Inhibition of maintenance DNA methylation by Stella. Biochem Biophys. Res. Commun. 453, 455-460 (2014).

10. Amouroux, R. et al. De novo DNA methylation drives $5 \mathrm{hmC}$ accumulation in mouse zygotes. Nat. Cell Biol. 18, 225-233 (2016).

11. Li, Y. et al. Stella safeguards the oocyte methylome by preventing de novo methylation mediated by DNMT1. Nature 564, 136-140 (2018). 